\title{
An Improved Method of Hough Transform for Circle Recognition Based on Optimized Gradient
}

\author{
$\mathrm{He} \mathrm{Kui}^{1, \mathrm{a})}$, Hu YP ${ }^{2, \mathrm{~b})}$ \\ ${ }^{1}$ Dalian University of Technology, DaLian 116024, LiaoNing, China. \\ ${ }^{2}$ Dalian University of Technology, DaLian 116024, LiaoNing, China. \\ a)18940874616@163.com \\ b)hypok@dlut.deu.cn
}

Keywords: Hough transform, Circle recognition, Optimized gradient.

\begin{abstract}
For the situation in which the low accuracy of circular hole recognition in hole workpiece based on image processing technology, a method of Optimized Gradient Hough Transform(OGHT) for circle recognition is proposed. Based on the Standard Hough Transform (SHT), the OGHT is used to extract the edge feature firstly, then select a certain number of edge pixels to evaluate the gradient to determine the center of circle. Next, calculating and sorting the coincidence degree between the rounds recognized by Standard Hough Transform and the edge in source image, and finally the highest rank is identified as the circle we needed. The proposed method is verified by comparative experiments, which improves the accuracy of circle recognition with little effect on efficiency.
\end{abstract}

\section{INTRODUCTION}

Hough Transform is a mature algorithm, which is used for precise positioning of circles in image processing. Because of its good anti-noise performance and insensitivity to the phenomenon of partial cover, it is widely used in the fields of pattern recognition, machine version and artificial intelligence $^{[1]}$. Although SHT has strong robustness in anti-noise and anti-distortion, the method has the following disadvantages in practical application ${ }^{[2]}$ : (1) The SHT can only detect geometric graphic element with strict mathematical expressions, such as lines, circles and ellipses; (2) With the increase of parameters in the curve expression, both the complexity and the memory cost of the SHT are exponentially growing;(3) If we want to improve the accuracy of the recognition of the characteristic circle, more detailed parameter quantization is needed, which will lead to much more processing time and memory consumption; (4) There is a great error in the recognition if the radius is not properly controlled.

In order to overcome the shortcomings, many scholars propose a series of modified algorithms based on SHT, such as Generalized Hough Transform, Probabilistic Hough Transform, Fuzzy Hough Transform and Gradient Hough Transform. D.H.Ballard proposed the Generalization of Hough Transform[3], which can map from simple primitives to complex primitive. This makes the SHT a general transform that can be used to find any complex shape; On the basis of SHT, a Dynamic Generalized Hough Transform[4] is proposed by V.F.Leavers. By using of the information of each discrete point in this method, the accuracy of recognition is improved to a certain extent; N.Kiryati[5] has analyzed and verified that , during the final voting stage to determine the circle, the computational complexity is significantly reduced in using Hough Transform based on probability; XuLei[6] proposed another mature algorithm based on Probabilistic Hough Transform: Random Hough transform; James R Bergen[7] presented an algorithm of Hough Transform based on Monte Carlo method. Compared with the SHT algorithm, these methods improve the running efficiency and reduce the memory cost to a large extent.

Joon H. Han[8] presented the Fuzzy Hough Transform which aims to detect the characteristic shapes in the noise data; In 2001, C. Galambos[9] put forward a Gradient-based Hough Transform on the basis of the Probabilistic Hough Transform; on the shoulder of C.Galambos, a method of Hough Transform based on optimized gradient is proposed and the experimental results have shown that the accuracy of recognition was improved. 


\section{THEORETICAL BASIS OF HOUGH TRANSFORM FOR CIRCLE RECOGNITION}

The principle of Hough Transform for circle recognition is to use the duality of the dot-line to map the pixel of the image space into the parameter space firstly. And then all the points in the accumulator of parameter space will be cumulated for evaluation. Finally, according to the calculated value, we can determine the center and radius of the circle[10]. In the image space, the mathematical expression of the circle is shown in equation (1)

$$
\left(x_{i}-x_{0}\right)^{2}+\left(y_{i}-y_{0}\right)^{2}=r^{2} \quad \mathrm{i}=(1,2,3, \ldots, \mathrm{n})
$$

Whereas $\left(x_{0}, y_{0}\right)$ is the center coordinate, $\mathrm{r}$ is the radius, and $\left(x_{i}, y_{i}\right)$ is the coordinates of the circle. Hough transform is convert the circle of the image space into the parameter space. The mathematical expression of the circle in the parameter space is shown in equation (2)

$$
\left(x_{0}-x_{i}\right)^{2}+\left(y_{0}-y_{i}\right)^{2}=r^{2}
$$

The corresponding relationship between the parameter space and the image space is shown in the following Figure1

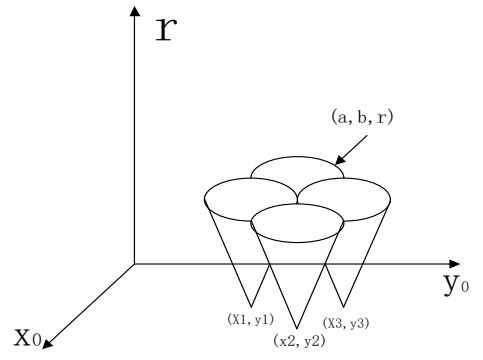

Figure1. The parameter space of Hough Transforms

When using the Hough Transform to determine the center coordinates and radius of the circle in the source image, a three-dimensional accumulator $A\left(x_{0}, y_{0}, r\right)$ needed to be created in the parameter space, where $r$ is a variable. Each pixel on the circle in the source image is accumulated by the formula (2), then calculating the maximum value $A_{\max }\left(x_{0}, y_{0}, r\right)$ in the accumulator $A\left(x_{0}, y_{0}, r\right)$. In this case, $\left(x_{0}, y_{0}\right)$ is the center coordinate and $\mathrm{r}$ is the radius of the circle in the image space.

\section{GRADIENT-BASED HOUGH TRANSFORM OF CIRCLE RECOGNITION}

The Gradient-based Hough Transform of circle recognition is based on the improvement of the Probabilistic Hough transform. On the basis of Probabilistic Hough Transform, two significant improvements have been put forward from the use of gradient. One is to limit the range of angular when calculating the gradient of edge pixel, which will reduce the running time and the response of the output to the noises. Second, the gradient value is used when the characteristic circle is determined at the end of the probabilistic Hough transform. These modifications improve the accuracy of the center positioning compared to the Probabilistic Hough Transform. But it is impossible to ensure the accuracy of the final recognition of the circle because there is no verification of the coincidence of the source image. Figure2 shows the experimental results of the Gradient-Based Hough Transform for circle recognition, in which the red circle is exactly recognized by this method. 


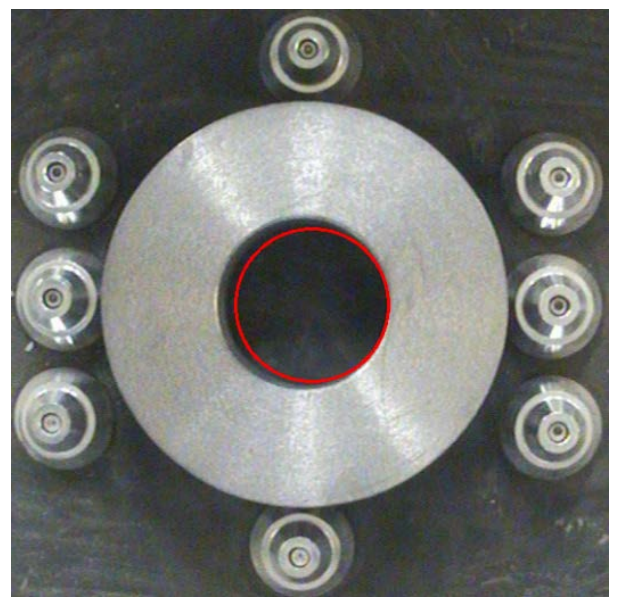

Figure2. The result of Gradient-Based Hough transform of circle recognition

In the figure2, it is necessary to identify the middle hole of the workpiece. The algorithm has some errors due to that the coincidence is not verified. When using the Gradient-Based Hough transform to identify the circle, the accurate positioning of the circle that requires to use a certain number of edge pixel gradient value. Because of the noises and the errors of gradient calculation, it is very easy to cause the error of positioning. Thus there is no guarantee of accuracy without subsequent verification of coincidence. In this paper, the OGHT introduces the concept of coincidence $\Theta$ which rules out the circles with a big difference between radius, and improves the accuracy of circle recognition to a certain extent.

\section{THE METHOD OF OPTIMIZED GRADIENT HOUGH TRANSFORM FOR CIRCLE RECOGNITION}

This paper improves the accuracy of the Standard Hough Transform for circle recognition by optimizing the function of HoughCircles in OpenCV[11]. The HoughCircles use the voting value of edge pixel gradient to determine the center position and radius of the characteristic circle. However, there is a certain error in the calculation of the edge pixel gradient, which may even lead to the deviation of more than 30 degrees in the actual direction of gradient. If you do not have some constraints on the radius of the circle, it is difficult to accurately find the ideal characteristic circle. In this paper, on the basis of the Gradient-based Hough Transform of circle recognition, the coincidence between the series of characteristic circle and source image contours is calculated and sorted, and then the top ranked circle will be selected as what we need. The algorithm flow shown in Figure3 


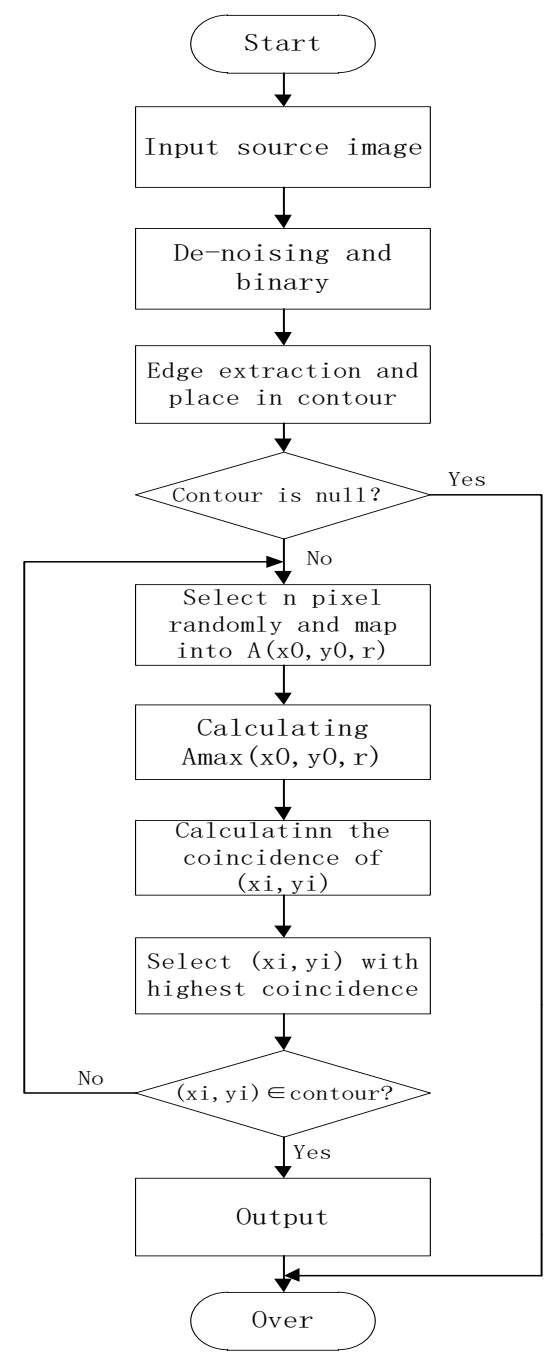

Figure3. The optical gradient algorithm flow diagram

The key to this algorithm is the calculation of the coincidence. Generally, the shorter distance between the two centers, or the smaller difference between the radius, the higher the coincidence is. Therefore, the coincidence is affected by the center distance $d_{o}$ and the radius difference $d_{r}$ between the two circles. In order to facilitate subsequent sort processing, the mathematical expression of coincidence $\Theta$ is as follows:

$$
\theta=\frac{1}{d_{o}+d_{r}}
$$

Whereas $d_{o}$ represents the center distance between the two circles, while $d_{r}$ is the difference between the radius. In this paper, the center point $\left(x_{0}, y_{0}\right)$ and the radius $r$ of the image space are determined by the parameter space $A_{\max }\left(x_{0}, y_{0}, r\right)$.To reduce the amount of calculating, the radius of these circles that are greater or less than a given radius of $1 / 10$ are ruled out firstly, and then the coincidence $\Theta$ is calculated for the remaining circle according to the formula (3). Using this algorithm to determine the characteristic circle, the center of the circle is determined by the gradient voting and the radius is constrained by the coincidence $\Theta$, so the accuracy is significantly improved. The experimental result of the algorithm is shown in Figure 4 and the red circle is identified by this method: 


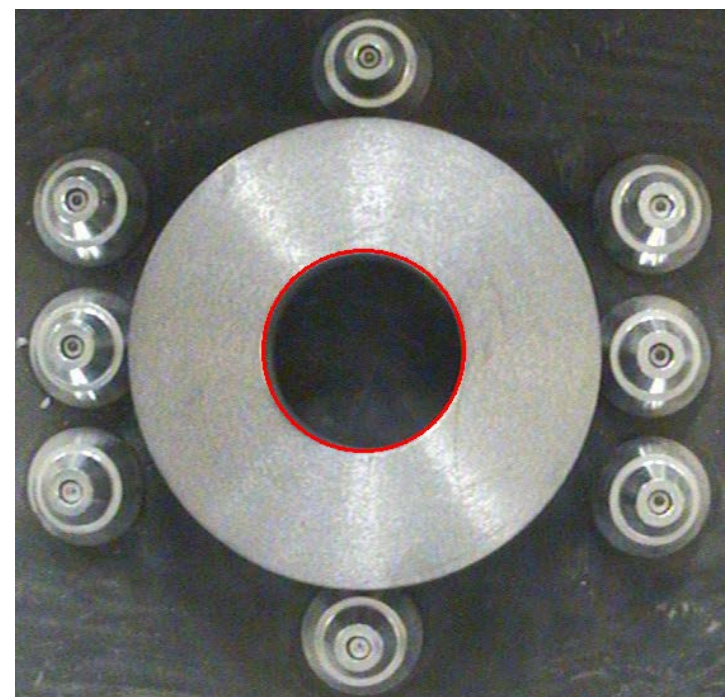

Figure4. The result of Optimized-Gradient Hough transform of circle recognition

\section{ANALYSIS OF EXPERIMENTAL RESULTS}

Table 1 shows the data comparison between SHT, GHT and OGHT in running time: TABLE 1. Three method Runtime Comparison

\begin{tabular}{|c|c|c|c|c|c|c|c|c|c|c|}
\hline & 1 & 2 & 3 & 4 & 5 & 6 & 7 & 8 & 9 & 10 \\
\hline SHT & 2113 & 2241 & 2532 & 2196 & 2064 & 2305 & 2292 & 3027 & 1925 & 2583 \\
\hline GHT & 853 & 728 & 956 & 762 & 884 & 917 & 698 & 1023 & 768 & 819 \\
\hline OGHT & 1021 & 1039 & 1295 & 831 & 1056 & 1137 & 899 & 1197 & 921 & 1096 \\
\hline
\end{tabular}

Runningtime: ms

According to the definition of coincidence $\Theta$ of formula (3), we know that the coincidence indirectly reflects the accuracy of recognition, and the experimental results also prove its correctness. On the other hand, the table (1) data analysis shows that the calculation of coincidence makes the running time increased slightly at the same time. Since the data in image space is projected into the parameter space, the edge pixels is first identified by edge extraction. Then a certain number of edge pixels are randomly selected into the accumulator, which greatly improves the efficiency.

\section{CONCLUSION}

In this paper, we analyze the key steps of SHT, GHT and OGHT for circle recognition, and find out the accuracy of the OSHT method has improved. Although the OSHT algorithm is relatively insufficient in running time, the accuracy of its recognition is more obvious than that of the other. In the specific industrial production applications, such as industrial inspection, which needs higher accuracy of recognition, the method of OGHT has better effect to achieve the prearranged aim.

\section{REFERENCES}

[1]. Wu M, Song Z, Li B, et al. A Method to Detect Circle based on Hough Transform[C]. Citeseer, 2015.

[2]. Mukhopadhyay P, Chaudhuri B B. A survey of Hough Transform[J]. Pattern Recognition. 2015, 48(3): 993-1010. 
[3]. Ballard D H. Generalizing the Hough transform to detect arbitrary shapes[J]. Pattern Recognition. 1991, 13(2): 183-194.

[4]. Leavers V F. Active intelligent vision using the dynamic generalized Hough Transform.[C]. 1990.

[5]. Kiryati N, Eldar Y, Bruckstein A M. A probabilistic Hough transform[J]. Pattern Recognition. 1991, Volume 24: 303-316.

[6]. Xu L, Oja E, Kultanen P. A new curve detection method: Randomized Hough transform[J]. Pattern Recognition Letters. 2009, Volume 11, Issue 5: 331-338.

[7]. Bergen J R, Shvaytser H. A probabilistic algorithm for computing Hough transforms[J]. Journal of Algorithms and Computational Technology. 1991, Volume 12: 639-656.

[8]. Han J H, Kóczy L, Poston T. Fuzzy Hough transform[J]. Pattern Recognition Letters. 1994, Volume 15: 649-658.

[9]. Galambos C, Kittler J, Matas J. Gradient based progressive probabilistic Hough transform[J]. IEE Proceedings - Vision, Image, and Signal Processing. 2001, 148(3): 158.

[10]. Yadav V K, Batham S, Acharya A K, et al. Approach to accurate circle detection: circular hough transform and local maxima concept[C]. IEEE, 2014.

[11]. Open C V. OpenCV documentation[Z]. Obtingut de http://opencv. org/documentation. html, 2013. 